\title{
Gewerkschaften und andere Akteure der Arbeitszeitpolitik - Wer bestimmt über die Zeit?
}

Die zentrale Rolle der Gewerkschaften bei der Durchsetzung von Arbeitszeitregulierung war lange Zeit unumstritten. Sie gründete sich auf eine erfolgreiche Politik der Arbeitszeitverkürzung, die sich fast über das gesamte 20. Jahrhundert erstreckte. Im Gefolge der Arbeitszeitflexibilisierung geriet die Arbeitszeitpolitik der Gewerkschaften in eine Krise. Doch werden in der Krise vielleicht auch Ansatzpunkte für eine Revitalisierung der Arbeitszeitpolitik im Interesse der Arbeitnehmerinnen und Arbeitnehmer sichtbar? Welche Rolle können dabei Beschäftigte und Betriebsräte spielen und was würde dies für die Arbeitszeitpolitik der Gewerkschaften bedeuten?

\section{Einleitung}

Arbeitszeitregulierung ist ein wesentlicher Beitrag zur „Dekommodifizierung“ des Beschäftigungsverhältnisses. Mit ihrer Hilfe soll verhindert werden, dass die Dauer, aber auch die Lage und Verteilung der Arbeitszeit, zum bloßen Anhängsel der Konkurrenzerfordernisse werden, denen die Unternehmen ausgesetzt sind. Historisch ist dies durch die Reduzierung der Arbeitszeiten pro Tag, Woche und Jahr ermöglicht worden. Der Hauptmotor, der immer wieder periodische Kompromisse mit den Unternehmen über die Abgrenzung von Arbeitszeit und Freizeit erzwungen hat, waren über mehr als ein Jahrhundert hinweg die Gewerkschaften. Sicher, ohne die Beschäftigten, ohne die vielen Mitglieder und, wenn es darauf ankam, Streikenden hätten die Gewerkschaften nie die Kraft gehabt, die Arbeitszeitverkürzungen durchzusetzen. Aber die Tarifverträge, in denen definiert wird, wer wie lange (und vielleicht auch: wann) über die Zeit der abhängig Beschäftigten bestimmt, schließen die Gewerkschaften ab. Insofern bestimmen sie über die Zeit der Beschäftigten mit. Die Frage, wer Arbeitszeitpolitik macht, entscheidet darüber, wer über Zeit bestimmt oder zumindest mitbestimmt.

Diese „Mitbestimmung“ ist nicht mehr selbstverständlich. In den 1990er Jahren ist in Deutschland die Initiative in der Arbeitszeitpolitik mehr und mehr auf die Unternehmen übergegangen. Der Schwerpunkt verlagerte sich zunächst auf die Flexibilisierung der Arbeitszeiten im Betrieb. Der Betrieb ist auch der Dreh- und Angel- punkt, über den Unternehmerverbände seit einigen Jahren versuchen, die Arbeitszeiten wieder zu verlängern. An kollektive Arbeitszeitverkürzungen, die stets wie ein Vitaminstoß für Arbeitszeitregulierung gewirkt haben, wagt heute kaum jemand zu denken.

Die Rekommodifizierung, der die verschiedenen Seiten des Beschäftigungsverhältnisses gegenwärtig unterworfen werden (Bosch 2001), hat längst auch die Arbeitszeit erfasst. Die Zeit wird wieder stärker vom Arbeitgeber bestimmt. Das historisch erreichte Modell des Einflusses von Gewerkschaften auf die Arbeitszeiten ist zwar noch nicht überrollt, wird aber teilweise unterlaufen, und es scheint gelegentlich ins Leere zu greifen. Die Frage „Wer bestimmt über die Zeit?" stellt sich neu. Mit ihr setzen wir uns im Folgenden auseinander, und damit zugleich mit den Akteuren, also denjenigen, die Arbeitszeitpolitik machen. Wir gehen dabei in drei Schritten vor: Nach einem Rückblick auf den traditionellen Arbeitszeitkompromiss beleuchten wir die Hauptfelder seiner aktuellen Krise. Abschließend richten wir den Blick auf die unterschiedlichen Akteure, von denen heute eine Revitalisierung der Arbeitszeitpolitik ausgehen könnte.

\section{Zeitkompromiss und gewerkschaftliche Gegenmacht}

Die gewerkschaftliche "Mitbestimmung“ über die Zeit konnte über Jahrzehnte hinweg darauf bauen, dass auch die Unternehmen Vorteile in der - nicht auf ihr eigenes
Betreiben zurückgehenden - Dekommodifizierung des Beschäftigungsverhältnisses und Begrenzung der Arbeitszeit zu nutzen gelernt haben. Die gewerkschaftlichen Erfolge in der Arbeitszeitregulierung schlossen zumindest in der Bundesrepublik Deutschland stets einen Produktivitätskompromiss mit den Arbeitgebern ein: Arbeitszeitverkürzungen seien auf wirtschaftliches Wachstum und steigende Produktivität der Unternehmen zu gründen (Deutschmann et al. 1987). Indem immer wieder aufs Neue die Claims über die Zeit abgesteckt und damit für die Unternehmen die Türen zu Kostensenkungen durch Arbeitszeitverlängerungen versperrt wurden, erwiesen sich die Arbeitszeitverkürzungen als „Rationalisierungspeitsche“ und als Treiber für innovations- und qualitätsorientierte Produktmarktstrategien.

Diese Kompromisskonstellation zwischen Gewerkschaften und Arbeitgebern war allerdings - neben den politischen Kräfteverhältnissen - an zumindest zwei wichtige wirtschaftliche Voraussetzungen

Thomas Haipeter, Dr., Institut Arbeit und Qualifikation (IAQ), Universität DuisburgEssen. Arbeitsschwerpunkte: Industrielle Beziehungen, Industriesoziologie. e-mail: thomas.haipeter@uni-due.de Steffen Lehndorff, Dr., Leiter der Forschungsabteilung Arbeitszeit und Arbeitsorganisation am Institut Arbeit und Qualifikation (IAQ), Universität Duisburg-Essen. Arbeitsschwerpunkte: Arbeitsorganisation, Arbeitszeiten, Beschäftigungsstrukturen und Arbeitsbeziehungen im internationalen Vergleich.

e-mail: steffen.lehndorff@uni-due.de 
gebunden: auf der einen Seite ein kontinuierliches Wirtschaftswachstum, wie es die Phase des Wirtschaftswunders kennzeichnete, und auf der anderen Seite Produktionsmodelle zahlreicher Unternehmen, die an den Prinzipien standardisierter Massenproduktion ausgerichtet waren und deshalb ohnehin auf der Abkoppelung der Produktions- von der Marktökonomie und der zeitökonomischen Rationalisierung der Arbeit beruhten.

Dass die wirtschaftlichen und politischen Geschäftsgrundlagen dieses Zeitkompromisses sich in den zurückliegenden zwei Jahrzehnten wesentlich geändert haben und wie dies das Gefüge der Arbeitszeitregulierung in Deutschland erschüttert, ist auch von uns bereits ausführlich analysiert worden (Haipeter/Lehndorff 2004; Lehndorff 2006). Im vorliegenden Aufsatz konzentrieren wir uns auf die politische Seite dieses Umbruchs.

In der Arbeitszeitpolitik hatte sich, entsprechend der korporatistischen Regulierungsstrukturen des westdeutschen Systems der industriellen Beziehungen, eine Hierarchie der Gestaltungsebenen und damit eine Hierarchie der Arbeitszeitakteure eingespielt. An der Spitze standen die Gewerkschaftszentralen als Takt- und Impulsgeber der Arbeitszeitpolitik gegenüber der Arbeitgeber-Tarifpartei. Die Betriebsräte hatten hingegen die Aufgabe, sich auf die Organisation und Kontrolle der Umsetzung der Arbeitszeitnormen im Betrieb und auf die Verhandlung von Abweichungen, seien es bezahlte Mehrarbeit, Kurzarbeit oder später dann Gleitzeitregelungen, zu konzentrieren. Die Beschäftigten schließlich waren, abgesehen von den Phasen des offenen Tarifkonflikts, insofern mehr Objekte als Subjekte der Arbeitszeitpolitik, als sie durch die kollektiven Vereinbarungen geschützt wurden. Individuelle Arbeitszeitgestaltung innerhalb standardisiert regulierter Arbeitszeiten geschah in der Regel informell.

Bereits die Einführung der Gleitzeit in den Angestelltenbereichen passte nicht so recht in die Hierarchie der Gestaltungsebenen und war deshalb seitens der Gewerkschaften zunächst nicht Gegenstand von Gestaltung, sondern Gegenstand von Misstrauen. Die Gewerkschafts- und Betriebsratspolitik war traditionell auf die machtpolitisch ausschlaggebende Klientel orientiert: die männlichen Industriearbeiter. Weder Angestellte noch Frauen, die ab den 1960er Jahren immer zahlreicher und vor- rangig als Teilzeitbeschäftigte den Arbeitsmarkt betraten, wurden als gesonderte Interessengruppen wirklich wichtig genommen.

Die wirtschaftlichen Grundlagen dieser klassischen Arbeitszeitpolitik wurden in den 1980er Jahren zwar schwächer, doch mit einer in Europa beispiellosen Kraftanstrengung, dem Streik für den Einstieg in die 35-Stunden-Woche, konnten zumindest Teile der bundesdeutschen Gewerkschaften ihre Gegenmacht im Zeitkonflikt, ihre Mit-Bestimmung über die Zeit, zunächst verteidigen. Auch der Mitgliederrückgang, unter dem viele Gewerkschaften anderer westlicher Länder bereits zu leiden hatten, konnte vorerst aufgehalten werden. Doch der große Erfolg von 1984 ging mit neuen Herausforderungen einher. Spätestens in den 1990er Jahren wurde das Problem offensichtlich: Die „Verbetrieblichung" der Arbeitszeitpolitik (Schmidt/ Trinczek 1999) stellte sowohl die Hierarchie der Regulierungsebenen infrage als auch das politische Machtgefüge, das ihr entsprach. Heute lässt sich die Arbeitszeitregulierung als eine zerklüftete Landschaft beschreiben. In ihr sind drei große Problemfelder auszumachen, die wir im Folgenden in aller Kürze umreißen.

\section{Krisenregionen der Arbeitszeitregulierung}

Wie wirksam Arbeitszeitregulierung ist, lässt sich am einfachsten an der Dauer der „normalerweise“, also tatsächlich im Durchschnitt gearbeiteten Wochenstunden von Vollzeitbeschäftigten ablesen. Betrachtet man diesen Basis-Indikator, so ist - aus der Sicht von Gewerkschaften - die Lage besser als die Stimmung. Die gewöhnliche Wochenarbeitszeit von Vollzeitbeschäftigten, wie sie vom Mikrozensus bzw. der Europäischen Arbeitskräftestichprobe ausgewiesen wird, liegt in Ost- wie in Westdeutschland bei rund 40 Stunden und damit ungefähr im EU-Durchschnitt (vgl. dazu und zum Folgenden Lehndorff/Wagner 2004a). Hinter den Durchschnittszahlen verbergen sich aber Ausdifferenzierungen (Seifert 2007): Männer arbeiten länger als Frauen, Angestellte länger als Arbeiter, und beide Unterschiede werden selbst bei Vollzeitbeschäftigten allmählich größer. Die stärkste Ausdifferenzierung jedoch ist die zwischen Vollzeit und Teilzeit. Insbesondere durch den Boom der Minijobs sinkt die durchschnittliche Arbeitszeit von Teilzeitbeschäftigten, und diese sind in der großen Mehrzahl Frauen.

Sehen wir uns also die zerklüftete Landschaft der Arbeitszeitregulierung an, die Hauptfelder ihrer Krise. Diese Problembereiche - oder „Länder“ der Arbeitszeitregulierung, wie wir sie bezeichnen wollen - lassen sich jeweils am Beispiel bestimmter Beschäftigtengruppen besonders deutlich herausarbeiten. Dies bedeutet jedoch nicht, dass nicht auch andere Beschäftigtengruppen von diesen Problemen betroffen wären. Ganz im Gegenteil: In der betrieblichen Wirklichkeit treten die Krisenfelder der Arbeitszeitregulierung häufig gebündelt und geballt in Erscheinung.

\subsection{DAS STAMMLAND}

Beginnen wir mit den Bereichen, in denen die Gewerkschaften mithilfe der Flächentarifverträge die tatsächlichen Arbeitszeiten nach wie vor unmittelbar beeinflussen. Hier liegt das Stammland der Arbeitszeitregulierung. Wie die Entwicklung spätestens seit 2004 zeigt, werden gerade hier die großen Konflikte um die Dauer der Arbeitszeit ausgetragen.

Zum Stammland der Arbeitszeitregulierung gehören vor allem die größeren Betriebe der Industrie: Hier sind die Arbeitszeiten am kürzesten, und hier sind sie am stärksten (aber auch am flexibelsten) reguliert (Lehndorff/Wagner 2004a; Bauer et al. 2004). Die Tarifbindung ist überdurchschnittlich hoch, und Betriebsräte und Gewerkschaften sind besonders einflussreich. Die Großbetriebe der Industrie sind so etwas wie die Flaggschiffe des Tarifvertragssystems. Erschütterungen im Stammland zeigen Wirkungen in vielen anderen Bereichen. Nicht allein bei den mittelgroßen Betrieben derselben Branchen, die verständlicherweise für sich einfordern, was den mächtigen Konzernen zugestanden wird, sondern ebenso in Dienstleistungsbranchen wie dem Einzelhandel oder der Deutschen Bahn. In enger Wechselwirkung mit dem politischen Klimawandel in den Hochburgen der deutschen Exportwirtschaft stand insbesondere der Angriff der Landesregierungen auf Arbeitszeiten und Tarifordnung des öffentlichen Dienstes.

Was sich in den vergangenen Jahren dramatisch verändert hat, ist vor allem die Infragestellung der traditionellen Kompro- 
missstruktur der „Konfliktpartnerschaft“ durch Teile der Arbeitgeberverbände, die sich durch die wechselseitige Anerkennung von Interessen auszeichnete (MüllerJentsch 1999). Mit Verweis auf Renditeforderungen, Auslagerungsdruck oder Standortkonkurrenz werden Betriebsräte und Gewerkschaften zu Konzessionen bei der Reduzierung von Arbeitsstandards gerade auch in der Arbeitszeit gedrängt, die die Prägekraft der Flächentarifverträge auszuhebeln drohen. Der Versuch, mit Hilfe des Pforzheimer Abkommens Abweichungen vom Flächentarifvertrag $\mathrm{zu}$ regulieren, ist eine Reaktion auf den sich ausbreitenden informellen Wildwuchs, häufig als „betriebliche Bündnisse" drapiert, und auf die nachlassende Tarifbindung der Unternehmen (Haipeter/Schilling 2006; Kohaut/Schnabel 2006). ${ }^{1}$ Unternehmen, die heute darauf verzichten, sich einem Arbeitgeberverband anzuschließen oder die gar aus ihm austreten, haben in vielen Fällen nur eine geringe Gegenwehr von Gewerkschaften zu befürchten. Die Schwäche der Arbeitgeberverbände ist daher die Kehrseite einer wachsenden organisationsund betriebspolitischen Schwäche der Gewerkschaften. Der Anteil der in DGB-Mitgliedsgewerkschaften organisierten abhängig Beschäftigten ist zwischen 1995 und 2004 von etwa $29 \%$ auf nur noch $18 \%$ abgesackt - dies ist der zweitstärkste Rückgang unter den Ländern der EU-15, der den Organisationsgrad der Gewerkschaften in Deutschland auf Rang 17 innerhalb der erweiterten EU zurückgeworfen hat (Kohl et al. 2006).

In den Auseinandersetzungen der vergangenen Jahre lässt sich eine bedeutsame Veränderung der tarifpolitischen Landschaft beobachten. Während früher der Satz galt, dass durch Tarifverträge die Konflikte eine Zeit lang stillgelegt wurden, lebt ein Tarifvertrag heute davon, dass er von der Konfliktbereitschaft der Beschäftigten in den Betrieben gestützt wird. Über einen längeren Zeitraum hinweg haben die Gewerkschaften bei vitalen Themen wie der Arbeitszeitpolitik keinen betriebspolitischen Gestaltungsanspruch mehr angemeldet. Dies zu ändern, ist ein Ziel der neuerlichen Hinwendung zur gewerkschaftlichen Betriebspolitik insbesondere in der Metallindustrie. Wenn es den Gewerkschaften damit gelingt, für die Beschäftigten im Betrieb wieder unmittelbar präsent zu werden, wird sich dies zugleich vorteilhaft auf die Erschließung des Neulandes der Arbeitszeitregulierung auswirken, dem wir uns jetzt zuwenden.

\subsection{NEULAND}

Im Neuland der Arbeitszeitregulierung haben Tarifverträge nur vermittelt oder abgeschwächt - wenn überhaupt - Spuren in den Arbeitszeitrealitäten hinterlassen. Am offensichtlichsten ist diese Situation dort, wo hoch qualifizierte Angestellte arbeiten. Sie haben traditionell längere Arbeitszeiten als andere Beschäftigtengruppen, wofür in der Angestelltenforschung unter anderem eine höhere Karriere- und Beitragsorientierung der Angestellten verantwortlich gemacht wird (Kotthoff 1997). Zugleich fallen dort neue Steuerungsformen, die auf die Eigeninitiative der Beschäftigten zur Erreichung der Unternehmensziele setzen, auf einen besonders fruchtbaren Boden (vgl. am Beispiel der Zeitungsredakteure: Haipeter 2004 und den Beitrag von Kratzer/Sauer in diesem Heft). Zwar ließe sich einwenden, dass höher und hoch qualifizierte Angestellte für die Zukunft der Arbeitszeitregulierung keine ausschlaggebende Bedeutung haben werden. Das Problem ist aber, dass diese Beschäftigtengruppen eine Vorreiterfunktion für neue Formen der Arbeitsorganisation ausüben, die von dort auf unterschiedliche Bereiche der Arbeitswelt ausstrahlen, sodass sie sich zukünftig auf die Arbeitszeit anderer großer Teile der Beschäftigten auswirken können (Lehndorff 2006).

Seit Beginn der 1990er Jahre wird in vielen Unternehmen eine Koppelung der Produktion von Gütern und Dienstleistungen an Marktschwankungen durchgesetzt. Zugleich werden Unternehmensstrategien und Geschäftsfeldstrukturen stärker an Finanzmarkterwartungen ausgerichtet. Outsourcing, Standortkonkurrenz und Leiharbeit werden systematisch als Mittel der Konfrontation der Beschäftigten mit der Konkurrenz auf Güter- und Arbeitsmärkten genutzt. Damit verbunden ist sowohl eine restriktive Personalpolitik als auch ein Druck auf Interessenvertretungen und Kollektivverträge. Die Beschäftigten müssen sich die Sicherheit ihres Arbeitsplatzes täglich aufs Neue erarbeiten, und sie tun dies am besten in eigener Initiative. Die restriktive Personalpolitik behindert den Abbau von Arbeitszeitsalden wegen fehlender Vertretungsmöglichkeiten, und die Konfrontation von Beschäftigten mit Konkurrenz auf dem Güter- und Arbeitsmarkt kann eine
Haltung fördern, bestehende Arbeitszeitregelungen in eigener Regie zu unterlaufen, um den Anforderungen gerecht werden zu können.

Die Veränderungen der Arbeitsorganisation hinterlassen bereits Spuren bei den Arbeitszeiten. Betrachtet man die Beschäftigten mit überdurchschnittlich langen Arbeitszeiten, dann springt die enge Verknüpfung von Selbststeuerung der Arbeitszeiten und Leistungsdruck ins Auge. So liegen bei einem Drittel der Beschäftigten, die ihre täglichen Anwesenheitszeiten selbst steuern, die tatsächlichen Wochenarbeitszeiten um mehr als vier Stunden über der vertraglichen Arbeitszeit. Zugleich geben Beschäftigte mit langen Arbeitszeiten besonders häufig an, ständig unter Zeitdruck $\mathrm{zu}$ arbeiten (Bauer et al. 2004, S. 99ff.). In einer von ver.di durchgeführten Befragung erklärten $68 \%$ der Antwortenden, dass Ausmaß und Tempo ihrer Arbeit vor allem von den direkten Anforderungen der Kunden bzw. anderer Abteilungen abhingen; an zweiter und dritter Stelle wurden selbst gesteckte Ziele (42\%) und Vorgaben der Vorgesetzten (37\%) genannt (Lehndorff/Wagner 2004b). Wenn Leistungssteuerung über die Konfrontation mit Kundenanforderungen ein derartiges Gewicht bekommt und dies auf betriebliche Milieus mit traditionell schwacher Arbeitszeitregulierung trifft, dann sind die Rahmenbedingungen für Arbeitszeitverlängerung in eigener Initiative besonders günstig.

\subsection{NIEMANDSLAND}

Nehmen wir nun einen Perspektivenwechsel vor: Die Veränderungen der Arbeitszeiten von Frauen im Lebenslauf markieren ein Feld, das bislang weitgehend unbeeinflusst von Arbeitszeitregulierung geblieben ist. Im Unterschied zu Männern ist die Entwicklung von Arbeitszeiten und Einkommen von Frauen entscheidend durch die Existenz (und die Zahl) von Kindern geprägt. Deutschland gehört zu den Ländern, in denen die Geburt von Kindern eine

\footnotetext{
Zwar gibt es im Rahmen tariflicher Öffnungsklauseln seit Jahren bereits zahlreiche Betriebsvereinbarungen über kürzere Arbeitszeiten, doch bei den Abweichungen vom Tarifvertrag auf der Basis des "Pforzheimer Abkommens" geht es, wenn Arbeitszeit eine Rolle spielt (was bei rund $60 \%$ der über 900 bis Ende 2006 abgeschlossenen Ergänzungstarifverträge der Fall ist), um Arbeitszeitverlängerungen.
} 
Reduzierung des Arbeitsangebotes von Frauen auslöst, sei es in Form eines mittelbis langfristigen Rückzugs aus der Erwerbstätigkeit oder in Form eines Verbleibs in Teilzeit nach der „Kinderpause“ (Anxo et al. 2006). Das Dilemma besteht darin, eine „Entlastung der ,rush hour of life" " (Klammer 2005) zu erreichen, ohne auf berufliche Entwicklungsmöglichkeiten verzichten zu müssen. Frauen, die sich - ähnlich wie Männer - beruflich entwickeln möchten, werden häufig vor die harte Wahl zwischen Kind und eigenständiger Erwerbstätigkeit gestellt.

Selbstverständlich drückt sich hier vor allem die rückständige Struktur des Steuerund Sozialversicherungssystems und der „Familienpolitik“ in Deutschland aus, die gegenwärtig - mit einer Verzögerung von über 30 Jahren gegenüber nordeuropäischen Ländern - vor allem am Thema Kinderbetreuung ins Zentrum der öffentlichen Aufmerksamkeit gerät (vgl. dazu den Beitrag von Heitkötter/Lange in diesem Heft). In einer geradezu symbiotischen Beziehung zu diesen konservativen Rahmenbedingungen befindet sich die vorherrschende Arbeitszeitrealität. Der bevorzugte Wiedereinstieg für Frauen nach mehrjähriger "Kinderpause" ist die Teilzeitarbeit, der Weg zur Fortsetzung der beruflichen Karriere im Rahmen einer Vollzeittätigkeit bleibt zumeist versperrt (Beckmann 2002; O'Reilly/Bothfeld 2002).

Wenn Frauen, die sich in Erwerbstätigkeit befinden, nach ihren Arbeitszeitwünschen befragt werden, stehen Arbeitszeiten, die sie im Sinne lebensphasenbezogener Wechsel zwischen Voll- und Teilzeit ihrer Lebenssituation anpassen können, ganz oben auf ihrer Wunschliste (Klenner 2005). Insgesamt tendieren die Arbeitszeitwünsche von Frauen am stärksten - in Deutschland ähnlich wie in einigen anderen EULändern - zur 30-Stunden-Woche (Bielenski et al. 2002). Die 30-Stunden-Woche, oder „kurze Vollzeit“ (Spitzley 2006), wird stark nachgefragt, aber wenig angeboten. In der Tarifpolitik spielt sie bislang keine Rolle. Es fehlen also sowohl im Betrieb als auch in den sozialstaatlichen Rahmenbedingungen noch weitgehend die materiellen Voraussetzungen für eine tatsächliche Entscheidungsfreiheit über die variable Gestaltung der Lebensarbeitszeit von Frauen und Männern.

Ziehen wir eine kurze Zwischenbilanz. Die Charakterisierung der Krisenregionen führte bislang zu drei Schlussfolgerungen:
Erstens: Tarifverträge leben heute davon, dass sie von der Konfliktbereitschaft der Beschäftigten in den Betrieben getragen werden. Sie müssen aber - zweitens - nicht allein von Kollektiven verteidigt, sondern auch von einzelnen Beschäftigten als nützlich erkannt werden. Und drittens ist Arbeitszeitpolitik ein sowohl über die Tarifpolitik als auch die Betriebspolitik hinausreichendes Feld. All dies hat weitreichende Konsequenzen für die Frage, wer zukünftig Arbeitszeitpolitik machen muss, wenn Zeit nicht immer einseitiger von Unternehmensinteressen bestimmt werden soll.

\section{Neue Akteurskonstella- tionen: Gewerkschaften und...}

Die Gewerkschaften haben ihre Initiative in der Arbeitszeitpolitik verloren. Welche Chancen bieten sich ihnen, verlorenes Terrain zurückzuerobern? Der Ausgangsgedanke für die Beantwortung dieser Frage ist, so meinen wir, dass eine veränderte Arbeitswelt eine veränderte Arbeitszeitregulierung benötigt, und diese von einem neuen Zusammenspiel verschiedener arbeitszeitpolitischer Akteure getragen sein wird. Sehen wir uns die möglichen Akteure der Reihe nach an.

\section{1 ...UNTERNEHMEN}

Von Unternehmensseite geht seit den 1990er Jahren in Deutschland die stärkste arbeitszeitpolitische Initiative aus. Waren früher die Gewerkschaften die Vorkämpfer der Arbeitszeitpolitik mit dem Leitthema der Arbeitszeitverkürzung, so sind es jetzt die Unternehmen mit Leitthemen wie Flexibilisierung oder Arbeitszeitverlängerung. Weitblickende Manager nehmen jedoch auch Gestaltungsthemen wie „work-lifebalance" oder variable Lebensarbeitszeiten in den Blick (Riedmann et al. 2006). Häufig mag dies zunächst nicht mehr sein als ein programmatischer Anspruch, doch es wäre grundverkehrt, dies als modisches Wortgeklingel abzutun. Insbesondere bei den zuletzt genannten Gestaltungsthemen sind neuartige betriebliche und überbetriebliche Koalitionen vorstellbar, in denen zugleich ein wertvolles Druckpotenzial gegenüber der Bildungs- und Familienpolitik des Staates liegen kann.
Dabei ist jedoch zu beachten, dass Unternehmen zwar Akteure der Arbeitszeitpolitik sein können, aber keine Triebkräfte der Arbeitszeitregulierung sind. Das eigenständige Interesse von Unternehmen an Kollektivverträgen beschränkt sich auf deren Ordnungs- und Befriedungsfunktion und auf die Vermeidung von Such- und Verhandlungskosten. Doch aus diesen Interessen allein entstehen noch keine Tarifverträge. Diese gibt es nur, weil die Beschäftigten ein starkes Interesse an deren Schutz-, Partizipations- oder Verteilungsfunktion haben (Bispinck/Schulten 1998). Wenn kollektive Arbeitszeitregulierungen existieren, dann können Unternehmen durchaus von ihnen profitieren, doch entstanden sind sie bislang nur im Konflikt mit den Unternehmen. Es ist nicht ersichtlich, warum sich dies in Zukunft ändern sollte.

Allerdings stellt die verstärkte Initiative von Unternehmen in der Arbeitszeitpolitik eine Herausforderung an die Gewerkschaften dar, sich ebenfalls auf betrieblicher Ebene stärker zu engagieren. Die Notwendigkeit, eine aktive gewerkschaftliche Betriebspolitik zu entwickeln, ist mittlerweile unstrittig. Insbesondere in der IG Metall gibt es dazu verschiedene Ansätze mit interessanten Erfahrungen sowohl hinsichtlich einer möglichen Stärkung der gewerkschaftlichen Verhandlungsposition als auch einer möglichen Verbesserung der Mitgliederrekrutierung (Huber et al. 2006).

\section{2 ...BESCHÄFTIGTE}

So wie Arbeitszeitregulierung stets ein wesentlicher Beitrag zur Dekommodifizierung des Beschäftigungsverhältnisses war, ist die gegenwärtige Schwächung der Arbeitszeitregulierung ein Bestandteil der großen Rekommodifizierungs-Tendenz. Eine neuerliche Umkehr dieser Tendenz würde ganz sicherlich nicht einfach aufs Neue die traditionellen Formen des Schutzes der Beschäftigten vor der ungepufferten Wirkung der Konkurrenz auf dem Güter- und Arbeitsmarkt annehmen können. Um es auf einen einfachen Nenner zu bringen: Der Schutz der Beschäftigten würde in stärkerem Maße, als dies in den traditionellen Regulierungsformen möglich und intendiert war, Instrumente zum Selbstschutz beinhalten.

Die Einhaltung oder Erneuerung kollektivvertraglichen Schutzes erfordert heute auf der einen Seite in vielen Fällen ein wesentlich intensiveres betriebliches Enga- 
gement, als dies in traditionellen Tarifbewegungen üblich war. Der äußerste Ausdruck dieses Zwangs zu gewerkschaftlicher „Basisarbeit“ sind die sogenannten „organizing"-Kampagnen in Branchen mit besonders schlechten Arbeitsbedingungen und geringem gewerkschaftlichen Organisationsgrad. Es wäre ein fundamentaler Irrtum zu glauben, hier gehe es um Mobilisierung von „Kollektiven“: Im Mittelpunkt derartiger Kampagnen stehen die einzelnen Beschäftigten.

Auf der anderen Seite provozieren neue Formen der Arbeitsorganisation den Versuch, den einzelnen Beschäftigten mehr Möglichkeiten zu eröffnen, zu Hauptpersonen in der Arbeitszeitregulierung zu werden und ihre unterschiedlichen Lebenslagen und individuellen Interessen in die Arbeitszeitgestaltung einfließen zu lassen. Wenn das Management die Verantwortung der Einzelnen für den Erfolg des Unternehmens ins Zentrum rückt, dann gilt es, die darin mitschwingende Partizipationsverheißung konkret werden zu lassen. In der Praxis stehen Autonomie und Partizipation jedoch häufig nur auf dem Papier. Die Probleme kommen aus zwei grundverschiedenen Richtungen (Haipeter 2006): Teilweise ist eine Rückkehr der Hierarchie zu beobachten, bei der Partizipationsnormen der Arbeitszeitregulierung von den unteren Führungskräften für weitgehend unwirksam erklärt werden. Demgegenüber ziehen sich bei einer konsequenten Marktsteuerung, die bislang vor allem in Angestelltenbereichen anzutreffen ist, die Vorgesetzten häufig aus der Verantwortung für die Arbeitszeit weitgehend zurück mit der Begründung, dass die Beschäftigten selbst für die Einhaltung der Arbeitszeitnormen und die Rückführung überschüssiger Arbeitszeiten zu sorgen hätten.

Partizipative Arbeitszeitgestaltung widerspricht diesen beiden gegensätzlichen Strömungen gleichermaßen. Sie zielt darauf ab, dass Beschäftigte an der Gestaltung ihrer Arbeitsbedingungen in Aushandlungen mit ihren Führungskräften beteiligt sind, also nicht allein "Handlungsautonomie“, sondern auch "Verhandlungsautonomie“ haben (Moldaschl 2001, S. 136). Letztlich stehen dabei die Rahmenbedingungen zur Debatte, die für mögliche Restriktionen der Handlungsautonomie sorgen.

Kollektivverträge können durchaus so gestaltet werden, dass sie derartig prozessbezogene Normen enthalten, die dem individuellen Interessenhandeln der Beschäf- tigten Ansatzpunkte bieten (Boes/Trinks 2006). Wir nennen derartige Normen „Haltegriffe“, denn die Beschäftigten müssen selber nach ihnen greifen, und kein wohlmeinender Betriebsrat oder Gewerkschaftsfunktionär kann ihnen das abnehmen. Die praktischen Erfahrungen mit „Haltegriffen" sind höchst unterschiedlich, teilweise sogar gegensätzlich (Haipeter/ Lehndorff 2004). Das ausschlaggebende Erfolgskriterium scheint uns nicht in den objektiven Rahmenbedingungen, sondern im Umgang der betrieblichen Akteure und insbesondere des Betriebsrats mit der Regulierung zu liegen. Für die Stärkung der Verhandlungsautonomie der Beschäftigten unter den Bedingungen von Marktsteuerung wird nicht allein ein neuer Typ von Kollektivverträgen mit neuen Prozessnormen für Beschäftigte und ihre Interessenvertretungen benötigt, sondern auch - und vor allem - ein neuer Umgang mit ihnen. Zur Debatte steht hier letztlich das Selbstverständnis von Betriebsräten.

\section{3 ....BETRIEBSRÄTE}

Betriebsräte sind die „Anker“ der Arbeitszeitregulierung im Betrieb, weil es eine Vielzahl vitaler Funktionen der Arbeitszeitpraxis gibt, die nur sie ausfüllen können. Dazu gehört zunächst die Gegenmachtfunktion der Aushandlung von Betriebsvereinbarungen zur Arbeitszeit mit starken Autonomie- und Partizipationsrechten für die Beschäftigten, und mit starken „Mitbestimmungsschwellen“ für ihr eigenes Arbeitszeithandeln. Teil der Gegenmachtfunktion ist auch die Lösung von Arbeitszeitproblemen im Konflikt mit dem Management. Eine wichtige Voraussetzung für eine wirksame Gegenmacht ist aber auch, dass die Betriebsräte eine Gestaltungsmacht mit eigenen arbeitszeitpolitischen Konzepten sind. Und schließlich müssen sie als Ordnungsmacht in dem Sinne fungieren, dass sie einen Überblick über die Arbeitszeitentwicklungen behalten und bei Fehlverläufen korrigierend eingreifen. In einer Situation, in der Beschäftigte ihre individuellen Arbeitszeitinteressen artikulieren, wird die „Ordnungsmacht" aber nur dann entwickelt werden können, wenn die Betriebsräte die Beschäftigten als Experten in eigener Sache respektieren, sie in der Wahrung individueller Autonomie- und Partizipationsansprüche gegenüber dem Management unterstützen, ihnen also Hilfe zur Selbsthilfe anbieten.
Eine solche-zweifellos anspruchsvolle - Form der Interessenvertretung, bei der konfliktfähiges Co-Management mit einer ausgeprägten Partizipationsorientierung verbunden ist, lässt sich als partizipationsorientiertes Interessenmanagement beschreiben (vgl. zur Typologie von Interessenvertretungen unter den Bedingungen flexibler Arbeitszeitgestaltung: Haipeter 2006). Interessant an den sicherlich derzeit nicht weit verbreiteten „partizipationsorientierten Interessenmanagern" ist vor allem, dass ihnen das von den Erfahrungen der Partizipation geprägte Selbstverständnis der Beschäftigten einen neuartigen Rückhalt gibt. Gerade im Neuland der Arbeitszeitregulierung werden solche neuen Erfahrungen der Interessenvertretung durch Betriebsräte gesammelt, die durchaus auch Anregungen für die starken Betriebsräte des Stammlandes bieten. Doch $\mathrm{zu}$ einer Massenerscheinung kann diese schwierige Neuorientierung erst dann werden, wenn der kräftepolitische Hintergrund dies begünstigt. Wichtige Voraussetzungen dafür sind die Hilfe der Gewerkschaften, der institutionelle Hintergrund des Betriebsverfassungsgesetzes und Tarifverträge. Gute tarifliche Leitplanken im Sinne materieller und prozeduraler Mindeststandards sind wiederum wichtige Voraussetzungen für gute Betriebsvereinbarungen zur Arbeitszeit mit starken Haltegriffen für die Beschäftigten und wirksamen Mitbestimmungsschwellen für die Betriebsräte.

Vor dem Hintergrund der oben beschriebenen Hinwendung der Gewerkschaften zur Betriebspolitik ist dies nicht mehr gleichbedeutend mit der klassischen Aufgabenverteilung zwischen Betriebsrat und Gewerkschaft, die häufig als Arbeitsteilung zwischen verschiedenen Ebenen der Politik verstanden wurde - der betrieblichen Ebene einerseits, der Verantwortung für die Flächentarifverträge andererseits. Das veränderte, den Betrieb wieder stärker ins Zentrum rückende Selbstverständnis von Gewerkschaftspolitik schließt - ähnlich wie bei den Betriebsräten - ein Ernstnehmen der individuellen Beschäftigteninteressen ein. Im Unterschied zu den Betriebsräten jedoch sind Gewerkschaften auf allen Ebenen gefordert: nicht allein im Betrieb, sondern ebenfalls in der ,großen Politik“. 


\section{4 ...POLITIK}

Die Menschen in Deutschland beobachten die Gewerkschaften mit wieder zunehmender Sympathie, trauen ihnen jedoch weniger Durchsetzungskraft denn je zu. Dies war die Quintessenz einer Allensbach-Studie aus dem vergangenen Jahr (Köcher 2006). Innerhalb von nur drei Jahren stieg der Anteil der Menschen, die sich einen stärkeren gewerkschaftlichen Einfluss auf die Politik wünschen, von $28 \%$ auf $44 \%$. Noch stärker aber stieg der Anteil derjenigen, die den Einfluss der Gewerkschaften auf die Regierungspolitik als gering einschätzen: von $36 \%$ auf $60 \%$. Die für diesen Erdrutsch, und zwar in seinen beiden Aspekten, einschneidende Erfahrung im betreffenden Dreijahres-Zeitraum von 2003 bis 2005 war die Verkündung und Durchsetzung der Agenda 2010.

Auch für die gewerkschaftliche Betriebspolitik auf dem Feld der Arbeitszeit ist diese Beobachtung äußerst relevant. Individuelle Gestaltungsmöglichkeiten der Arbeitszeit sind für die Mehrheit der abhängig Beschäftigten nur realisierbar, wenn sie Rückenstärkung durch kollektivvertragliche Normen erhalten - aber für immer mehr Beschäftigte ist demgegenüber die Rückenstärkung durch staatliche Politik vorrangig. Der Staat ist in Deutschland seit Langem ein sehr wichtiger Akteur in der Arbeitszeitpolitik. Dies drückt sich zum ei- nen in sozialen Schutzrechten wie dem Arbeitszeitgesetz aus - auch wenn Deutschland zur Minderheit der EU-Länder gehört, in denen die Arbeitswoche noch nicht gesetzlich auf 40 Stunden oder weniger begrenzt ist (Lehndorff 2001; eironline 2006). Der Staat agiert aber zum anderen auch und dies zum Teil noch wirksamer - auf indirekte Weise. So werden durch den Mangel an Kinderbetreuungsmöglichkeiten und durch die kostspielige Subventionierung der männlichen Hauptverdiener-Ehe sehr kurze Arbeitszeiten von Frauen begünstigt oder gar erzwungen.

Die Lösung dieser Probleme rückt in Deutschland erst dann näher, wenn eine bessere tarifvertragliche Gestaltung von Lebensarbeitszeit anknüpfen kann an einer veränderten staatlichen Politik. Der Vergleich mit einigen anderen europäischen Ländern zeigt, wie eng die Entwicklung von Frauenerwerbstätigkeit und Chancengleichheit im Beruf mit einer Strategie ,sozialer Investitionen“ des Wohlfahrtsstaates zusammenhängt (Esping-Andersen 2002). Zugleich muss nüchtern ausgesprochen werden, dass eine solche Strategie nicht finanzierbar ist, wenn gleichzeitig an der Subventionierung des traditionellen Familienmodells mit Hilfe des Ehegattensplittings, der Mitversicherung von nicht berufstätigen Ehefrauen und nicht zuletzt der Minijobs festgehalten wird. Wer nicht bereit ist, dieses Problem anzupacken, sollte über „flexible Lebensarbeitszeiten“ besser schweigen.

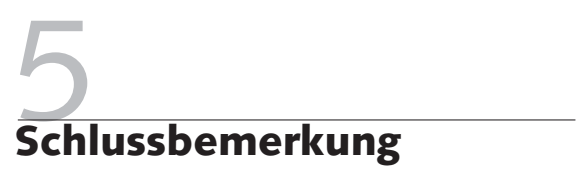

Arbeitszeitpolitik im Betrieb und in der Gesellschaft sind zwei Seiten einer Medaille. Arbeitszeitpolitik wird auf neue Weise politisch: Der Kampf um Autonomie und Partizipation im Arbeitsprozess ist ein letztlich politischer Konflikt um die Kontrolle innerhalb von Betrieben und Unternehmen; und der Konflikt um die Vereinbarkeit von Beruf und Kindern weitet die Grenzen industrieller Konflikte auf das Feld der Gesellschaftspolitik aus. Die Diskussion um Arbeitszeitpolitik kann sich deshalb nicht mehr auf kollektive Arbeitszeitregulierung allein konzentrieren, sondern umschließt zugleich die Frage der Organisation des Wohlfahrtsstaates und damit die entschieden politische Frage, wie in der Gesellschaft das Zusammenleben überhaupt gestaltet werden soll. Können die Gewerkschaften auf beiden Konfliktfeldern Erfolge erzielen, werden sie als Reformkraft der Arbeitszeitpolitik nicht nur den Charakter der industriellen Beziehungen nachhaltig verändern. 
Anxo, D./Boulin, J. Y./Fagan, C./Inmaculada, D./Keuzenkamp, S./ Klammer, U./Klenner, C./Moreno, G./Toharía, L. (2006): Working time options over the life course: New work patterns and company strategies. European Foundation for the Improvement of Living and Working Conditions, Luxembourg

Bauer, F./Groß, H./Lehmann, K./Munz, E. (2004): Arbeitszeit 2003. Arbeitszeitgestaltung, Arbeitsorganisation und Tätigkeitsprofile, Köln Beckmann, P. (2002): Zwischen Wunsch und Wirklichkeit - Tatsächliche und gewünschte Arbeitszeitmodelle von Frauen mit Kindern liegen noch immer weit auseinander. IAB-Werkstattbericht 12, Nürnberg Bielenski, H./Bosch, G./Wagner, A. (2002): Wie die Europäer arbeiten wollen. Erwerbs- und Arbeitszeitwünsche in 16 Ländern, Frankfurt/Main/ New York

Bispinck, R./Schulten, T. (1998): Globalisierung und das deutsche Tarifvertragssystem, in: WSI-Mitteilungen 4, S. 241-248

Boes, A./Trinks, K. (2006): "Theoretisch bin ich frei! " Interessenhandeln und Mitbestimmung in der IT-Industrie, Berlin

Bosch, G. (2001): Konturen eines neuen Normalarbeitsverhältnisses, in: WSI-Mitteilungen 4, S. 219-230

Deutschmann, C./Schmiede, R./Schudlich, E. (1987): Die langfristige Entwicklung der Arbeitszeit - Versuch einer sozialwissenschaftlichen Interpretation, Arbeitspapiere aus dem Arbeitskreis SAMF eironline (2006): Working time developments - 2005, http://eurofound. europa.eu/eiro/comparativestudies.html

Esping-Andersen, G. (2002) (with Gallie, D./Hemerick, A./Myles, J.): Why We Need a New Welfare State, Oxford

European Commission (2006): Employment in Europe 2006, Luxembourg

Haipeter, T. (2004): Zwischen den Zonen der Stabilität und Entkoppelung: Arbeitszeiten und Arbeitszeitregulierung bei den Zeitungsverlagen, in: Bsirske, F./Möning-Raane, M./Sterkel, G./Wiedemuth, J. (Hrsg.): Es ist Zeit: das Logbuch für die ver.di-Arbeitszeitinitiative, Hamburg, S. 110-154

Haipeter, T. (2006): Arbeits(zeit)politik zwischen Innovation und Eigensinn, in: Arbeit 15, S. 73-84

Haipeter, T./Lehndorff, S. (2004): Atmende Betriebe, atemlose Beschäftigte: Erfahrungen mit neuartigen Formen betrieblicher Arbeitszeitregulierung, Berlin

Haipeter, T./Schilling, G. (2006): Arbeitgeberverbände in der Metall- und Elektroindustrie: Tarifbindung, Organisationsentwicklung und Strategiebildung, Hamburg

Huber, B./Burkhard, O./Wagner, H. (Hrsg.) (2006): Perspektiven der Tarifpolitik. Im Spannungsfeld von Fläche und Betrieb, Hamburg Klammer, U. (2005): Flexicurity aus der Perspektive des Lebensverlaufs, in: Kronauer, M./Linne, G. (Hrsg.): Flexicurity. Die Bindung von Sicherheit an Flexibilität, Berlin

Klenner, C. (2005): Balance von Beruf und Familie - Ein Kriterium guter Arbeit, in: WSI-Mitteilungen 4, S. 207-213
Köcher, R. (2006): Mehr Zustimmung, aber weniger Zutrauen. Sympathiegewinne und Bedeutungsverluste der Gewerkschaften, in: FAZ vom 21. Juni, S. 5

Kohaut, S./Schnabel, C. (2006): Tarifliche Öffnungsklauseln: Verbreitung, Inanspruchnahme und Bedeutung, Diskussionspapiere des Lehrstuhls für VWL an der Friedrich-Alexander-Universität Erlangen-Nürnberg 41

Kohl, H./Lehndorff, S./Schief, S. (2006): Industrielle Beziehungen in Europa nach der Erweiterung, in: WSI-Mitteilungen 7, S. 403-409 Kotthoff, H. (1997): Führungskräfte im Wandel der Firmenkultur. QuasiUnternehmer oder Arbeitnehmer?, Berlin

Lehndorff, S. (2001): Weniger ist mehr. Arbeitszeitverkürzung als Gesellschaftspolitik, Hamburg

Lehndorff, S. (Hrsg.) (2006): Das Politische in der Arbeitspolitik. Ansatzpunkte für eine nachhaltige Arbeits- und Arbeitszeitgestaltung, Berlin Lehndorff, S./Wagner, A. (2004a): Arbeitszeiten und Arbeitszeitregulierung in Deutschland: eine Bestandsaufnahme, in: Bsirske, F./MöningRaane, M./Sterkel, G./Wiedemuth, J. (Hrsg.): Es ist Zeit: das Logbuch für die ver.di-Arbeitszeitinitiative, Hamburg, S. 40-72

Lehndorff, S./Wagner, A. (2004b), "Mein Engagement hängt von den

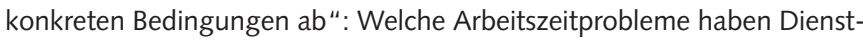
leistungsbeschäftigte, was erwarten sie von ver.di?, in: Bsirske, F./ Möning-Raane, M./Sterkel, G./Wiedemuth, J. (Hrsg.): Es ist Zeit: das Logbuch für die ver.di-Arbeitszeitinitiative, Hamburg, S. 195-236 Moldaschl, M. (2001): Herrschaft durch Autonomie - Dezentralisierung und widersprüchliche Arbeitsanforderungen, in: Lutz, B. (Hrsg.): Entwicklungsperspektiven von Arbeit, Weinheim, S. 132-164

Müller-Jentsch, W. (1999): Vorwort, in: ders. (Hrsg.): Konfliktpartnerschaft. Akteure und Institutionen der industriellen Beziehungen, München, S. 7-11

O'Reilly, J./Bothfeld, S. (2002): What happens after working part time? Integration, maintenance or exclusionary transitions in Britain and Western Germany, in: Cambridge Journal of Economics 26, pp. 409-439 Riedmann, A., (2006): (in cooperation with Bielenski, H./Szczurowska, T./Wagner, A.) Working time and work-life balance in European companies. Establishment Survey on Working Time 2004-2005. European Foundation for the Improvement of Living and Working Conditions, Luxembourg Schmidt, R./Trinczek, R. (1999): Der Betriebsrat als Akteur der industriellen Beziehungen, in: Müller-Jentsch, W. (Hrsg.): Konfliktpartnerschaft. Akteure und Institutionen der industriellen Beziehungen, München, S. 103-128

Seifert, H. (2007): Arbeitszeit - Entwicklungen und Konflikte, in: Aus Politik und Zeitgeschichte 4-5, S. 17-24

Spitzley, H. (2006): Solidarische Arbeitsverteilung und kurze Vollzeit, in: Siller, P./Dückert, T./Baumann, A. (Hrsg.): Neue Wege einer gerechten und emanzipativen Arbeitspolitik, Baden-Baden, S. 357-365 